\title{
Exponential convergence of $h p$-DGFEM for elliptic problems in polyhedral domains
}

\author{
D. Schötzau, C. Schwab, T. Wihler, M. Wirz
}

Abstract We review the recent results of $[21,22]$, and establish the exponential
convergence of $h p$-version discontinuous Galerkin finite element methods for the
numerical approximation of linear second-order elliptic boundary-value problems
with homogeneous Dirichlet boundary conditions and constant coefficients in three-
dimsional and axiparallel polyhedra. The exponential rates are confirmed in a series
of numerical tests.

\section{Introduction}

A key feature of the $h p$-version finite element method (FEM) is the possibility to achieve exponential convergence rates in terms of the number of degrees of freedom. Indeed, in the mid eighties, Babuška and Guo proved that using $h p$-FEM for the numerical approximation of elliptic boundary-value problems with piecewise analytic data in a polygonal domain $\Omega$ leads to energy norm error bounds of the $\infty$ form $C \exp \left(-b N^{1 / 3}\right)$, where $N$ is the dimension of the $h p$-version finite element space, and $C$ and $b$ are constants independent of $N$; see $[2,10,11]$ and the refer- ences therein. Exponential convergence is achieved by employing geometric mesh refinement towards the singular support $\mathscr{S}$ of the solutions (i.e., the set of vertices of $\Omega$ ), and nonuniform elemental polynomial degrees which increase linearly $r$

Dominik Schötzau

Department of Mathematics, University of British Columbia, Vancouver, BC, V6T 1Z2, Canada, e-mail: schoetzau@math.ubc.ca

Christoph Schwab

Seminar for Applied Mathematics, ETH, 8092 Zürich, Switzerland, e-mail: schwab@math.ethz.ch @̈

Thomas Wihler

Mathematisches Institut, Universität Bern, 3012 Bern, Switzerland, e-mail: wihler@math.unibe.ch ํ.

Marcel Wirz

Mathematisches Institut, Universität Bern, 3012 Bern, Switzerland, e-mail: wirz@ math.unibe.ch 
with the elements' distance from $\mathscr{S}$. The proof of elliptic regularity in countably weighted Sobolev spaces of the solutions, which constitutes an essential ingredient of the convergence proof, has been a major technical achievement. Let us mention that generalizations to conforming methods for higher-order elliptic problems and $h p$-version mixed methods for Stokes flow in polygons can be found in $[8,14,18]$.

In the nineties, steps to extend the analytic regularity and the $h p$-convergence analysis to polyhedral domains in three dimensions were undertaken in $[3,9,12,13]$ and the references therein. The difficulty in this case is the appearance of anisotropic edge and corner-edge singularities. It was claimed and confirmed numerically that the energy norm errors decay exponentially as $C \exp \left(-b N^{1 / 5}\right)$, i.e., with an exponent containing the fifth root of $N$.

The discontinuous Galerkin finite element method (DGFEM) emerged in the seventies as a stable discretization of first-order transport-dominated problems, and as a nonconforming discretization of second-order elliptic problems; see the historical survey [1] and the references therein. Later, in the nineties, DGFEM was employed to realize $h p$-version methods for first-order transport and for advectionreaction-diffusion problems in two- and three-dimensional domains (see $[15,16])$. Exponential convergence rates were established for piecewise analytic solutions excluding, in particular, corner singularities as occurring in polygonal domains. In the context of purely elliptic problems, the well-posedness of $h p$-version local discontinuous Galerkin methods was shown in [17]. Finally, exponential convergence of $h p$-DGFEM in polygonal domains was proved in [24] for diffusion problems, and in [23] for Stokes flow, thereby extending the results of Babuška and Guo to the discontinuous Galerkin framework. In the recent articles [21, 22], the $h p$-DGFEM for the approximation of three-dimensional elliptic problems in polyhedra was considered. In addition, the paper [25] addresses mixed $h p$-DGFEM discretizations of the linear elasticity and Stokes equations in polyhedral domains; this work is based on the inf-sup stability of mixed $h p$-DGFEM (based on uniform isotropic, but variable polynomial degrees) for our class of $h p$-discretizations, which has been established in $[19,20]$.

In this paper, we will review the recent results of [21, 22], and, in particular, the proof of exponential convergence with a fifth root in $N$ for an $h p$-version DGFEM for elliptic problems with constant coefficients in axiparallel polyhedra. Our proof is based on the recent analytic regularity results of [5], which measure corner, edge and corner-edge singularities in analytic classes of anisotropically weighted Sobolev spaces. We begin by introducing $h p$-version DG approximations on general meshes consisting of axiparallel and possibly anisotropic cuboids, along with elemental degree vectors which may also be anisotropic. Moreover, we review the wellposedness of the resulting finite element methods, show the Galerkin orthogonality property, and derive abstract error estimates for the DG energy errors. To resolve singularities, we shall then construct a family of anisotropically and geometrically refined meshes, characterized by a subdivision ratio $\sigma \in(0,1)$ and a number $\ell$ of refinements. The corresponding degree vectors are linearly increasing with slope $\mathfrak{s}>0$ away from corners and edges. This family of $h p$-discretizations contains, in particular, three-dimensional and anisotropic generalizations of all mesh-degree combina- 
tions which were found to be optimal in the univariate case in [7]. By proceeding as in [22], we will then specify suitable polynomial interpolation operators, and show that our estimates lead to the exponential convergence bound $C \exp \left(-b N^{1 / 5}\right)$ in the DG energy norm. We will also present a series of new numerical tests which verify the exponential convergence in three dimensions. In particular, we confirm the fifth root in $N$ for corner-edge singularities.

The outline of the article is as follows: In Section 2, we introduce a model problem, and recapitulate its analytic regularity in the weighted Sobolev spaces of [5]. In Section 3, we introduce and analyze an $h p$-version interior penalty DGFEM with anisotropic elemental polynomial degrees on meshes of anisotropic and axiparallel elements. Section 4 is devoted to proving our exponential convergence estimate on geometric mesh families. Finally, Section 5 contains a series of numerical results.

\section{Model problem and analytic regularity}

We consider the boundary-value problem

$$
\begin{array}{rlrl}
-\nabla \cdot(A \nabla u) & =f & & \text { in } \Omega \subset \mathbb{R}^{3}, \\
u=0 & & \text { on } \Gamma=\partial \Omega,
\end{array}
$$

where $\Omega$ is an axiparallel Lipschitz polyhedron, $A$ a constant symmetric positive definite coefficient matrix, and $f$ an analytic right-hand side (more precise assumptions will be made in Proposition 1 below).

We specify the precise regularity of the solution $u$ of (1)-(2) in countably normed weighted Sobolev spaces. To that end, we follow [5], but mention the papers [9, 12, 13] where alternative definitions of countably normed weighted Sobolev spaces in terms of local spherical coordinates have originally been defined and studied.

Let us denote by $\mathscr{C}$ the set of corners $c$, and by $\mathscr{E}$ the set of edges $e$ of $\Omega$. The singular support of the solution $u$ is given by

$$
\mathscr{S}=\left(\bigcup_{c \in \mathscr{C}} c\right) \cup\left(\bigcup_{e \in \mathscr{E}} e\right) \subset \Gamma .
$$

For $c \in \mathscr{C}, e \in \mathscr{E}$ and $x \in \Omega$, we define the distance functions:

$$
r_{c}(x)=\operatorname{dist}(x, c), \quad r_{e}(x)=\operatorname{dist}(x, e), \quad \rho_{c e}(x)=r_{e}(x) / r_{c}(x) .
$$

For each corner $c \in \mathscr{C}$, we define by $\mathscr{E}_{C}=\{e \in \mathscr{E}: c \cap \bar{e} \neq \emptyset\}$ the set of all edges of $\Omega$ which meet at $c$. For any $e \in \mathscr{E}$, the set of corners of $e$ is given by $\mathscr{C}_{e}=\{c \in \mathscr{C}: c \cap \bar{e} \neq \emptyset\}$. Then, for $c \in \mathscr{C}, e \in \mathscr{E}$ respecticely $e \in \mathscr{E}_{C}$, and a parameter $\varepsilon>0$, we define the neighborhoods 


$$
\begin{aligned}
\omega_{c} & =\left\{x \in \Omega: r_{c}(x)<\varepsilon \wedge \rho_{c e}(x)>\varepsilon \quad \forall e \in \mathscr{E}_{c}\right\}, \\
\omega_{e} & =\left\{x \in \Omega: r_{e}(x)<\varepsilon \wedge r_{c}(x)>\varepsilon \quad \forall c \in \mathscr{C}_{e}\right\}, \\
\omega_{c e} & =\left\{x \in \Omega: r_{c}(x)<\varepsilon \wedge \rho_{c e}(x)<\varepsilon\right\} .
\end{aligned}
$$

By choosing $\varepsilon$ sufficiently small, we may then partition the domain $\Omega$ into four disjoint parts,

$$
\bar{\Omega}=\overline{\Omega_{0} \dot{\cup} \Omega_{\mathscr{C}} \dot{\cup} \Omega_{\mathscr{E}} \dot{\cup} \Omega_{\mathscr{C} \mathscr{E}}},
$$

where

$$
\Omega_{\mathscr{C}}=\bigcup_{c \in \mathscr{C}} \omega_{c}, \quad \Omega_{\mathscr{E}}=\bigcup_{e \in \mathscr{E}} \omega_{e}, \quad \Omega_{\mathscr{C} \mathscr{E}}=\bigcup_{c \in \mathscr{C}} \bigcup_{e \in \mathscr{E}_{c}} \omega_{c e} .
$$

We shall refer to the subdomains $\Omega_{\mathscr{C}}, \Omega_{\mathscr{E}}$ and $\Omega_{\mathscr{C} \mathscr{E}}$ as corner, edge and corneredge neighborhoods of $\Omega$, respectively, and define the remaining interior part of the domain $\Omega$ by $\Omega_{0}:=\Omega \backslash \overline{\Omega_{\mathscr{C}} \cup \Omega_{\mathscr{E}} \cup \Omega_{\mathscr{C} \mathscr{E}}}$.

To each $c \in \mathscr{C}$ and $e \in \mathscr{E}$, we associate a corner and an edge exponent $\beta_{c}, \beta_{e} \in \mathbb{R}$, respectively. We collect these quantities in the multi-exponent

$$
\underline{\beta}=\left\{\beta_{c}: c \in \mathscr{C}\right\} \cup\left\{\beta_{e}: e \in \mathscr{E}\right\} \in \mathbb{R}^{|\mathscr{C}|+|\mathscr{E}|} .
$$

Inequalities of the form $\beta<1$ and expressions like $\beta \pm s$ are to be understood componentwise.

Near corners $c \in \mathscr{C}$ and edges $e \in \mathscr{E}$, we shall use local coordinate systems in $\omega_{e}$ and $\omega_{c e}$, which are chosen such that $e$ corresponds to the direction $(0,0,1)$. Then, we denote quantities that are transversal to $e$ by $(\cdot)^{\perp}$, and quantities parallel to $e$ by $(\cdot)^{\|}$. In particular, if $\alpha \in \mathbb{N}_{0}^{3}$ is a multi-index corresponding to the three local coordinate directions in $\omega_{e}$ or $\omega_{c e}$, then we have $\alpha=\left(\alpha^{\perp}, \alpha^{\|}\right)$, where $\alpha^{\perp}=\left(\alpha_{1}, \alpha_{2}\right)$ and $\alpha^{\|}=$ $\alpha_{3}$. Following [5, Definition 6.3], we introduce the anisotropically weighted seminorm

$$
\begin{aligned}
& |u|_{M_{\underline{\beta}}^{m}(\Omega)}^{2}=|u|_{H^{m}\left(\Omega_{0}\right)}^{2}+\sum_{e \in \mathscr{E}} \sum_{\substack{\alpha \in \mathbb{N}_{0}^{3} \\
|\alpha|=m}}\left\|r_{e}^{\beta_{e}+\left|\alpha^{\perp}\right|} \mathrm{D}^{\alpha} u\right\|_{L^{2}\left(\omega_{e}\right)}^{2} \\
& \quad+\sum_{c \in \mathscr{C}} \sum_{\substack{\alpha \in \mathbb{N}_{0}^{3} \\
|\alpha|=m}}\left(\left\|r_{c}^{\beta_{c}+|\alpha|} \mathrm{D}^{\alpha} u\right\|_{L^{2}\left(\omega_{c}\right)}^{2}+\sum_{e \in \mathscr{E}_{C}}\left\|r_{c}^{\beta_{c}+|\alpha|} \rho_{c e}^{\beta_{e}+\left|\alpha^{\perp}\right|} \mathrm{D}^{\alpha} u\right\|_{L^{2}\left(\omega_{c e}\right)}^{2}\right),
\end{aligned}
$$

for $m \in \mathbb{N}_{0}$, and define the norm $\|u\|_{M_{\underline{\beta}}^{m}(\Omega)}$ by $\|u\|_{M_{\underline{\beta}}^{m}(\Omega)}^{2}=\sum_{k=0}^{m}|u|_{M_{\underline{\beta}}^{k}(\Omega)}^{2}$. Here, $|u|_{H^{m}\left(\Omega_{0}\right)}^{2}$ is the usual Sobolev semi-norm of order $m$ on $\Omega_{0}$, and the operator $\mathrm{D}^{\alpha}$ denotes the partial derivative in the local coordinate directions corresponding to the multi-index $\alpha$. The space $M_{\underline{\beta}}^{m}(\Omega)$ is the weighted Sobolev space obtained as the closure of $C_{0}^{\infty}(\Omega)$ with respect to the norm $\|\cdot\|_{M_{\underline{\beta}}^{m}(\Omega)}$. Finally, for a weight $\underline{\gamma} \in$ $\mathbb{R}^{|\mathscr{E}|+|\mathscr{E}|}$, we define the analytic class 


$$
A_{\underline{\gamma}}(\Omega)=\left\{u \in \bigcap_{m \geq 0} M_{\underline{\gamma}}^{m}(\Omega): \exists C_{u}>0 \text { s.t. }|u|_{M_{\underline{\gamma}}^{m}(\Omega)} \leq C_{u}^{m+1} m ! \forall m \in \mathbb{N}_{0}\right\} .
$$

The following shift theorem from [5, Corollary 7.1] now establishes the analytic regularity of solutions to problem (1)-(2).

Proposition 1. There exist bounds $\beta_{\mathscr{E}}, \beta_{\mathscr{C}}>0$ (depending on $\Omega$ and the coefficients in (1)) such that, for all weight vectors $\beta$ satisfying

$$
0 \leq \beta_{e}<\beta_{\mathscr{E}}, \quad 0 \leq \beta_{c}<\frac{1}{2}+\beta_{\mathscr{C}}, \quad e \in \mathscr{E}, c \in \mathscr{C},
$$

the following property holds: if the right-hand side $f$ in (1) belongs to $A_{1-\underline{\beta}}(\Omega)$, then the solution $u$ of (1)-(2) belongs to $A_{-1-\beta}(\Omega)$.

\section{Discretization}

\subsection{Finite element spaces}

We consider (a family of) meshes $\mathscr{M}$ consisting of axiparallel cuboids $\{K\}$. Hence, each element $K$ is the image of the reference cube $\widehat{Q}=(-1,1)^{3}$ under a composition $\Phi_{K}: \widehat{Q} \rightarrow K$ of a translation and a dilation. We allow for anisotropic elements and irregular meshes. Additional assumptions will be introduced in (22) below. With each cuboid $K \in \mathscr{M}$, we associate a polynomial degree vector $\underline{p}_{K}=$ $\left(p_{K, 1}, p_{K, 2}, p_{K, 3}\right) \in \mathbb{N}^{3}$, whose components correspond to the coordinate directions in $\widehat{Q}=\Phi_{K}^{-1}(K)$. The polynomial degree is isotropic if $p_{K, 1}=p_{K, 2}=p_{K, 3}=p_{K}$. We combine the elemental polynomial degrees $\underline{p}_{K}$ into the polynomial degree vector $\underline{p}=\left\{\underline{p}_{K}: K \in \mathscr{M}\right\}$, and introduce the $h p$-version finite element space

$$
S^{\underline{p}}(\mathscr{M})=\left\{u \in L^{2}(\Omega):\left.u\right|_{K} \in \mathbb{Q}^{\underline{p}} \underline{x}_{K}(K), K \in \mathscr{M}\right\} .
$$

The local polynomial approximation space $\mathbb{Q}^{\underline{p}}{ }(K)$ is defined as follows: first, on the reference element $\widehat{Q}$ and for a polynomial degree vector $\underline{p}=\left(p_{1}, p_{2}, p_{3}\right) \in \mathbb{N}_{0}^{3}$, we introduce the tensor product polynomial space:

$$
\mathbb{Q}^{\underline{p}}(\widehat{Q})=\mathbb{P}^{p_{1}}(\widehat{I}) \otimes \mathbb{P}^{p_{2}}(\widehat{I}) \otimes \mathbb{P}^{p_{3}}(\widehat{I})=\operatorname{span}\left\{\widehat{x}_{1}^{\alpha_{1}} \widehat{x}_{2}^{\alpha_{2}} \widehat{x}_{3}^{\alpha_{3}}: \alpha_{i} \leq p_{i}, 1 \leq i \leq 3\right\} .
$$

Here, for $p \in \mathbb{N}_{0}$, we denote by $\mathbb{P}^{p}(\widehat{I})$ the space of all polynomials of degree at most $p$ on the reference interval $\widehat{I}=(-1,1)$. Then, if $K$ is an axiparallel element of $\mathscr{M}$ with associated elemental mapping $\Phi_{K}: \widehat{Q} \rightarrow K$ and polynomial degree vector $\underline{p}_{K}=\left(p_{K, 1}, p_{K, 2}, p_{K, 3}\right)$, we set

$$
\mathbb{Q}^{\underline{p}} \underline{p}_{K}(K)=\left\{u \in L^{2}(K):\left(\left.u\right|_{K} \circ \Phi_{K}\right) \in \mathbb{Q}^{\underline{p}} \underline{p}_{K}(\widehat{Q})\right\} .
$$


If the polynomial degrees are uniform and isotropic, i.e., $p_{K, 1}=p_{K, 2}=p_{K, 3}=p_{K}=$ $p \geq 1$ for all $K \in \mathscr{M}$, we simply write $S^{p}(\mathscr{M})$ instead of $S \underline{\underline{p}}(\mathscr{M})$.

\subsection{Element boundary operators}

We denote the set of all interior faces in $\mathscr{M}$ by $\mathscr{F}_{I}(\mathscr{M})$, and the set of all boundary faces by $\mathscr{F}_{B}(\mathscr{M})$. In addition, let $\mathscr{F}(\mathscr{M})=\mathscr{F}_{I}(\mathscr{M}) \cup \mathscr{F}_{B}(\mathscr{M})$ signify the set of all (smallest) faces of $\mathscr{M}$. Furthermore, for an element $K \in \mathscr{M}$, we denote the set of its faces by $\mathscr{F}_{K}=\{f \in \mathscr{F}: f \subset \partial K\}$. If $f \in \mathscr{F}_{K}$, then we denote by $h_{K, f}^{\perp}$ the diameter of $K$ perpendicular to the face $f$. Similarly, if $\underline{p}_{K}$ is the polynomial degree vector on $K$, we denote by $p_{K, f}^{\perp}$ the polynomial degree perpendicular to $f$.

Next, we recall the standard DG trace operators. For this purpose, consider an interior face $f=\partial K^{\sharp} \cap \partial K^{b} \in \mathscr{F}_{I}(\mathscr{M})$ shared by two neighboring elements $K^{\sharp}, K^{\natural} \in$ $\mathscr{M}$. Furthermore, let $v$ and $w$ be a scalar-valued function and a vector-valued function, respectively, both sufficiently smooth inside the elements $K^{\sharp}, K^{b}$. Then we define the following trace operators along $f$ :

$$
\llbracket v \rrbracket]=\left.v\right|_{K^{\sharp}} n_{K^{\sharp}}+\left.v\right|_{K^{\prime}} n_{K^{b}}, \quad\langle\langle w\rangle\rangle=1 / 2\left(\left.w\right|_{K^{\sharp}}+\left.w\right|_{K^{b}}\right) .
$$

Here, for an element $K \in \mathscr{M}$, we denote by $n_{K}$ the outward unit normal vector on $\partial K$. For a boundary face $f=\partial K \cap \partial \Omega \in \mathscr{F}_{B}(\mathscr{M})$ for $K \in \mathscr{M}$, and sufficiently smooth functions $v, w$ on $K$, we let $[v v]=\left.v\right|_{K} n_{\Omega},\langle\langle w\rangle\rangle=\left.w\right|_{K}$, where $n_{\Omega}$ is the outward unit normal vector on $\partial \Omega$.

\subsection{Discontinuous Galerkin discretizations}

For a given mesh $\mathscr{M}$ and associated polynomial degree distribution $\underline{p}$, we define the $h p$-version symmetric interior penalty DG solution $u_{\mathrm{D} G} \in S \underline{\underline{p}}(\mathscr{M})$ by

$$
u_{\mathrm{D} G} \in S^{\underline{p}}(\mathscr{M}): \quad a_{\mathrm{D} G}\left(u_{\mathrm{D} G}, v\right)=\int_{\Omega} f v \mathrm{~d} x \quad \forall v \in S^{\underline{p}}(\mathscr{M}),
$$

where the bilinear form $a_{\mathrm{D} G}(u, v)$ is given by

$$
\begin{aligned}
a_{\mathrm{D} G}(u, v)= & \left.\int_{\Omega}\left(A \nabla_{h} u\right) \cdot \nabla_{h} v \mathrm{~d} x-\int_{\mathscr{F}(\mathscr{M})}\left\langle\left\langle A \nabla_{h} v\right\rangle\right\rangle \cdot \llbracket u \rrbracket\right] \mathrm{d} s \\
& \left.-\int_{\mathscr{F}(\mathscr{M})}\left\langle\left\langle A \nabla_{h} u\right\rangle\right\rangle \cdot \llbracket v v\right] \mathrm{d} s+\gamma \int_{\mathscr{F}(\mathscr{M})} \mathrm{j}[\llbracket u] \cdot[[v] \mathrm{d} s .
\end{aligned}
$$

Here, $\nabla_{h}$ is the elementwise gradient, and $\gamma>0$ is the interior penalty parameter that will be chosen sufficiently large. Furthermore, $j \in L^{\infty}(\mathscr{F}(\mathscr{M}))$ is the face-wise constant function given by 


$$
\left.\mathfrak{j}\right|_{f}= \begin{cases}\frac{\max \left(p_{K_{\sharp}, f}^{\perp}, p_{K_{b}, f}^{\perp}\right)^{2}}{\min \left(h_{K_{\sharp}, f}^{\perp}, h_{K_{b}, f}^{\perp}\right)} & \text { if } f=\partial K_{\sharp} \cap \partial K_{b} \in \mathscr{F}_{I}(\mathscr{M}), \\ \frac{\left(p_{K, f}^{\perp}\right)^{2}}{h_{K, f}^{\perp}} & \text { if } f=\partial K \cap \partial \Omega \in \mathscr{F}_{B}(\mathscr{M}) .\end{cases}
$$

We remark that we have omitted an explicit dependence of the penalty jump terms on the diffusion tensor $A$.

\subsection{Well-posedness}

We show the well-posedness of the $h p$-DGFEM in the standard DG energy norm defined by

$$
\|v\|_{\text {DG }}^{2}=\int_{\Omega}\left|\nabla_{h} v\right|^{2} \mathrm{~d} x+\gamma \int_{\mathscr{F}} \mathfrak{j} \mid\left[\left.[v]\right|^{2} \mathrm{~d} s,\right.
$$

for any $v \in S^{\underline{p}}(\mathscr{M})+H^{1}(\Omega)$. To that end, we recall the anisotropic polynomial trace inequality from [21, Lemma 4.3 a)]: let $K=\left(0, h_{1}\right) \times\left(0, h_{2}\right) \times\left(0, h_{3}\right)$ be an axiparallel element, then there exists a constant $C_{I}>0$ only depending on the reference element such that

$$
\|q\|_{L^{2}(f)} \leq C_{I}\left(p_{K, f}^{\perp}\right)^{2}\left(h_{K, f}^{\perp}\right)^{-1}\|q\|_{L^{2}(K)}^{2}
$$

for all $f \in \mathscr{F}_{K}, K \in \mathscr{M}$, and $q \in \mathbb{Q}^{\underline{p}}{ }_{K}(K)$.

Proceeding as in [21, Theorem 4.4], the following result can be shown.

Proposition 2. There is a threshold parameter $\gamma_{\min }>0$ such that for $\gamma \geq \gamma_{\min }$ the $D G$ bilinear form $a_{\mathrm{D} G}(\cdot, \cdot)$ is continuous and coercive over $S^{\underline{p}}(\mathscr{M})$. That is, we have

$$
\begin{aligned}
\left|a_{\mathrm{DG}}(v, w)\right| & \leq C_{1}\|v\|_{\mathrm{DG}}\|w\|_{\mathrm{DG}} & & \forall v, w \in S^{\underline{p}}(\mathscr{M}), \\
a_{\mathrm{DG}}(v, v) & \geq C_{2}\|v\|_{\mathrm{DG}}^{2} & & \forall v \in S^{\underline{p}}(\mathscr{M}) .
\end{aligned}
$$

The constants $\gamma_{\min }, C_{1}$ and $C_{2}$ only depend on $\gamma$ appearing in (17) and (19), the coefficient matrix $A$, and the constant $C_{I}$ in the trace inequality (20).

Next, we discuss the Galerkin orthogonality of the DG scheme (16) under the assumption that the solution $u$ of (1)-(2) belongs to $M_{-1-\underline{\beta}}^{2}(\Omega)$ for a weight vector $\underline{\beta}$ as in (11). We notice that, in this case, it is not obvious that the expression $a_{\mathrm{D} G}(u, v)$ is well defined for $v \in S \underline{p}(\mathscr{M})$, since $u$ may exhibit corner and/or edge singularities. Here, integrals of the form

$$
\int_{f} A \nabla u \cdot v \mathrm{~d} s, \quad f \in \mathscr{F}_{K} \cap \mathscr{F}_{B}(\mathscr{M}),
$$


appearing in the bilinear form $a_{\mathrm{D} G}$ require some special care, in particular, for faces $f$ which abut at the singular support $\mathscr{S}$. However, in [21, Section 4.5], it is shown that the regularity $u \in M_{-1-\beta}^{2}(\Omega)$ implies $A \nabla u \in L^{1}(f), f \in \mathscr{F}_{K} \cap \mathscr{F}_{B}(\mathscr{M})$. Thus, the above integrals are in fact properly defined as bilinear forms on $L^{1}(f) \times$ $L^{\infty}(f)$. Consequently, a Green's formula can be established which leads to the following result; see [21, Theorem 4.9].

Proposition 3. Let the solution u of (1)-(2) satisfy $u \in M_{-1-\beta}^{2}(\Omega)$ for $\underline{\beta}$ as in (11), and let $u_{\mathrm{D} G}$ be the DG approximation of (16) obtained with $\gamma \geq \gamma_{\min }$ (cf. Proposition 2). Then we have the Galerkin orthogonality property $a_{\mathrm{D} G}\left(u-u_{\mathrm{D} G}, v\right)=0$ for all $v \in S \underline{p}(\mathscr{M})$.

\subsection{Error estimates}

To derive error estimates, we shall now assume the following bounded variation property in the mesh size: there is a constant $\lambda \in(0,1)$ such that

$$
\lambda \leq h_{K^{b}, f}^{\perp} / h_{K^{\sharp}, f}^{\perp} \leq \lambda^{-1},
$$

for all interior faces $f=\partial K^{\dagger} \cap K^{\sharp} \in \mathscr{F} I(\mathscr{M})$, uniformly in the mesh family.

To account for the singular solution behavior near corner and edges, we disjointly partition $\mathscr{M}$ into

$$
\mathscr{M}=\mathfrak{O} \cup \dot{T},
$$

where elements in $\mathfrak{O}$ are bounded away from $\mathscr{S}$, and elements in the terminal layer $\mathfrak{T}$ have a nontrivial intersection with the singular support $\mathscr{S}$.

Let now $u \in M_{-1-\beta}^{2}(\Omega)$ be the solution of problem (1)-(2), and $u_{\mathrm{D} G} \in S \underline{p}(\mathscr{M})$ be the DG approximation from (16). As usual we split the error into the two parts

$$
u-u_{\mathrm{D} G}=\eta+\xi, \quad \text { with } \eta=u-\Pi u \text { and } \xi=\Pi u-u_{\mathrm{D} G} \in S^{\underline{p}}(\mathscr{M}),
$$

for an appropriate $h p$-version (quasi)interpolation operator $\Pi u \in S \underline{p}(\mathscr{M})$ of $u$.

To bound $\|\xi\|_{\mathrm{DG}}$ in terms of quantities involving $\eta$, we apply the coercivity of the DG form in Proposition 2, the Galerkin orthogonality property in Proposition 3, and the anisotropic trace inequality: given a cuboid $K=\left(0, h_{1}\right) \times\left(0, h_{2}\right) \times\left(0, h_{3}\right)$, $v \in W^{1, t}(K)$ for $t \geq 1$, there exists a constant $C_{t}>0$ only depending on $t$ and the reference element such that

$$
\|v\|_{L^{t}(f)}^{t} \leq C_{t}\left(h_{K, f}^{\perp}\right)^{-1}\left(\|v\|_{L^{t}(K)}^{t}+\left(h_{K, f}^{\perp}\right)^{t}\left\|\mathrm{D}_{K, f, \perp} v\right\|_{L^{t}(K)}^{t}\right),
$$

for any $f \in \mathscr{F}_{K}$; cf. [21, Lemma 4.2]. Here, the operator $\mathrm{D}_{K, f, \perp}$ signifies the partial derivative on element $K$ in direction perpendicular to $f$.

Consequently, we find the following generic error bound; see [21, Theorem 4.10] for details. 
Theorem 1. Assume (22) and let $u \in M_{-1-\underline{\beta}}^{2}(\Omega)$ with $\underline{\beta}$ as in (11). Then we have the error estimate

$$
\left\|u-u_{\mathrm{DG}}\right\|_{\mathrm{DG}}^{2} \leq C|\underline{p}|^{4}\left(E_{\mathfrak{O}}[\eta]+E_{\mathfrak{T}}[\eta]\right),
$$

where

$$
\begin{aligned}
E_{\mathfrak{O}}[\eta]= & \sum_{K \in \mathfrak{O}}\left(\max _{f \in \mathscr{F}_{K}}\left(h_{K, f}^{\perp}\right)^{-2}\|\eta\|_{L^{2}(K)}^{2}+\|\mathrm{D} \eta\|_{L^{2}(K)}^{2}\right) \\
& +\sum_{K \in \mathfrak{O}} \sum_{f \in \mathscr{F}_{K}}\left(h_{K, f}^{\perp}\right)^{2}\left\|\mathrm{D}_{K, f, \perp} \mathrm{D} \eta\right\|_{L^{2}(K)}^{2},
\end{aligned}
$$

and

$$
\begin{aligned}
E_{\mathfrak{T}}[\eta]= & \sum_{K \in \mathfrak{T}}\left(\max _{f \in \mathscr{F}_{K}}\left(h_{K, f}^{\perp}\right)^{-2}\|\eta\|_{L^{2}(K)}^{2}+\|\mathrm{D} \eta\|_{L^{2}(K)}^{2}\right) \\
& +\sum_{K \in \mathfrak{T}} \sum_{f \in \mathscr{F}_{K}}|f|^{-1} h_{K, f}^{\perp}\|\mathrm{D} \eta\|_{L^{1}(f)}^{2} .
\end{aligned}
$$

The constant $C>0$ is independent of the elemental aspect ratios, mesh sizes, and polynomial degree vectors. The quantity $|f|$ is the surface measure of a face $f$, and $|p|=\max _{K \in \mathscr{M}} \max \left\{p_{K, 1}, p_{K, 2}, p_{K, 3}\right\}$ is the maximal polymial degree.

\section{Exponential convergence on $h p$-version subspaces}

\subsection{Geometric meshes}

To construct geometrically refined meshes, we start from a coarse regular and shaperegular, quasi-uniform partition $\mathscr{M}^{0}=\left\{Q_{j}\right\}_{j=1}^{J}$ of $\Omega$ into $J$ convex axiparallel hexahedra. Each of these elements $Q_{j} \in \mathscr{M}^{0}$ is the image under an affine mapping $G_{j}$ of the reference patch $\widetilde{Q}=(-1,1)^{3}$, i.e., $Q_{j}=G_{j}(\widetilde{Q})$. The mappings $G_{j}$ are again compositions of (isotropic) dilations and translations.

We then introduce three canonical geometric refinements towards corners, edges and corner-edges of $\widetilde{Q}$, which are referred to as extensions (Ex2), (Ex3), and (Ex4) in [21], and which are illustrated in Figure 1. The extension (Ex1) introduced in [21] corresponds to the case where no refinement is considered on $\widetilde{Q}$.

Geometric meshes in $\Omega$ are now obtained by applying the patch mappings $G_{j}$ to transform these canonical geometric mesh patches on the reference patch $\widetilde{Q}$ to the macro-elements $Q_{j} \in \mathscr{M}^{0}$. More precisely, we denote by $\widetilde{\mathscr{M}_{j}}=\{\widetilde{K}\}_{\widetilde{K} \in \widetilde{\mathscr{M}_{j}}}$ the elements in the canonical geometric mesh patch associated with $Q_{j} \in \mathscr{M}^{0}$. The patches $Q_{j}$ away from the singular support $\mathscr{S}$ (i.e., with $\bar{Q}_{j} \cap \mathscr{S}=\emptyset$ ) are left unre- 

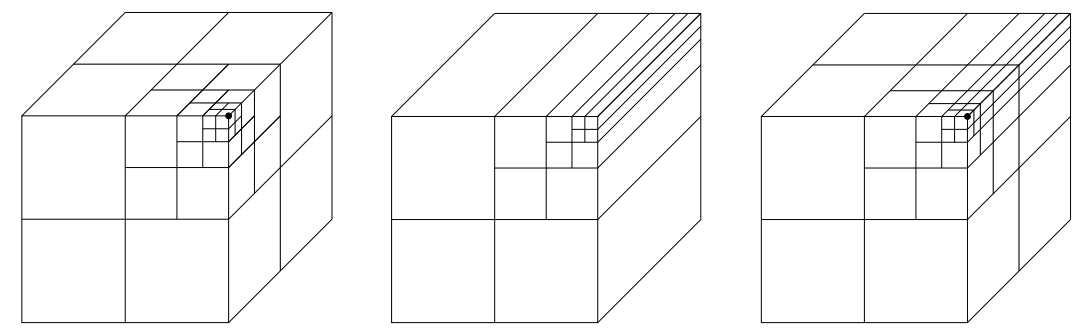

Fig. 1 Canical geometric refinements in $\widetilde{Q}$ with subdivision ratio $\sigma=\frac{1}{2}$. (Ex2): isotropic towards the corner $c$ (left), (Ex3): anisotropic towards the edge $e$ (center), (Ex4): anisotropic towards the edge-corner pair $c e$ (right). The sets $c, e, c e$ are shown in boldface.

fined by taking $\widetilde{\mathscr{M}_{j}}=\{\widetilde{Q}\}$. Then, we denote by $\mathscr{M}_{j}=\left\{K=G_{j}(\widetilde{K}): \widetilde{K} \in \widetilde{\mathscr{M}_{j}}\right\}$ the patch mesh on $Q_{j}$, and a geometric mesh in $\Omega$ is given by

$$
\mathscr{M}=\bigcup_{j=1}^{J} \mathscr{M}_{j}
$$

It is important to note that the geometric refinements in the canonical patches have to be suitably selected, oriented and combined in order to achieve a proper geometric refinement towards corners and edges of $\Omega$. By construction, each element $K \in \mathscr{M}$ is the image of the reference cube $\widehat{Q}=(-1,1)^{3}$ under an element mapping $\Phi_{K}=$ $G_{j(K)} \circ H_{K}: \widehat{Q} \rightarrow K \in \mathscr{M}$, where $H_{K}: \widehat{Q} \rightarrow \widetilde{K}, \widetilde{K} \subset \widetilde{M}_{j}$, is a possibly anisotropic dilation combined with a translation, and $G_{j}(K): \widetilde{Q} \rightarrow Q_{j}$ is the patch map.

In what follows, we will consider a sequence of $\sigma$-geometrically refined meshes denoted by $\mathfrak{M}_{\sigma}=\left\{\mathscr{M}_{\sigma}^{(\ell)}\right\}_{\ell \geq 1}$. Here, $\sigma \in(0,1)$ is a fixed parameter defining the ratio of the geometric subdivisions in the canonical refinements in Figure 1. The index $\ell$ is the refinement level. There holds: if $K \in \mathscr{M}_{\sigma}^{(\ell)}$, then there exists $K^{\prime} \in \mathscr{M}_{\sigma}^{(\ell-1)}$ such that $K \subset K^{\prime}$. We shall refer to the sequence $\mathfrak{M}_{\sigma}$ as a $\sigma$-geometric mesh family; see [21, Definition 3.4].

In addition to the mesh refinements, the extensions (Ex1)-(Ex4) in [21, Section 3] also provide appropriate polynomial degree distributions $\left\{p^{(\ell)}\right\}_{\ell \geq 1}$. They increase $\mathfrak{s}$-linearly away from the singular set $\mathscr{S}$. In particular, in the edge and corner-edge patches the polynomial degrees are anisotropic. On elements in the interior of the domain, they are uniform, isotropic and proportional to the number $\ell$ of geometric refinements.

Let us point out that the geometric mesh family constructed in [21, Section 3] satisfies the bounded variation property (22) with a constant $\lambda \in(0,1)$ depending on $\sigma$ and $\mathscr{M}^{0}$. In addition, the associated family of polynomial degree vectors $\left\{\underline{p}^{(\ell)}\right\}_{\ell \geq 1}$ satisfies a similar property in the polynomial degrees: there is a constant $\mu \in(0,1)$ depending on the slope parameter $\mathfrak{s}$ such that

$$
\mu \leq p_{K^{\natural}, f}^{\perp} / p_{K^{\sharp}, f}^{\perp} \leq \mu^{-1},
$$


for all interior faces $f=\partial K^{\dagger} \cap K^{\sharp} \in \mathscr{F}_{I}(\mathscr{M})$ and $\ell \geq 1$.

\subsection{Exponential convergence rates}

The main result of this review is the following exponential convergence result from [22, Theorem 6.1].

Theorem 2. Assume that the right-hand side $f$ of the boundary-value problem (1)(2) belongs to $A_{1-\underline{\beta}}(\Omega)$ for a weight vector $\underline{\beta}$ as in (11) (hence, the solution $u$ is in $A_{-1-\underline{\beta}}(\Omega)$ due to Proposition 1).

Let $\mathfrak{M}_{\sigma}=\left\{\mathscr{M}_{\sigma}^{(\ell)}\right\}_{\ell \geq 1}$ be a $\sigma$-geometric mesh family with a geometric refinement factor $\sigma \in(0,1)$ and $\left\{p^{(\ell)}\right\}_{\ell \geq 1}$ the associated (possibly anisotropic) $\mathfrak{s}$-linear degree distribution vectors with a slope parameter $\mathfrak{s}>0$, generated by the hp-extensions (Exl)-(Ex4) in [21, Section 3]. Consider the resulting hp-version finite element spaces

$$
V_{\sigma, \mathfrak{s}}^{(\ell)}:=S^{(\ell)}\left(\mathscr{M}_{\sigma}^{(\ell)}\right), \quad \ell \geq 1 .
$$

Then, for each $\ell \geq 1$, the DG approximation $u_{\mathrm{D} G} \in V_{\sigma, \mathfrak{s}}^{(\ell)}$ is well defined for $\gamma \geq \gamma_{\min }$ (see Proposition 2), and we have the error estimate

$$
\left\|u-u_{\mathrm{DG}}\right\|_{\mathrm{DG}} \leq C \exp \left(-b N^{1 / 5}\right),
$$

where $N=\operatorname{dim}\left(V_{\sigma, \mathfrak{s}}^{(\ell)}\right)$. The constants $C>0$ and $b>0$ are independent of $N$, and solely depend on the constants in the trace inequalities (20) and (25), respectively, the parameters $\sigma, \mathfrak{s}$, the initial mesh $\mathscr{M}^{0}$, the analyticity constant $C_{u}$ in (10) of the solution $u$, the weight vector $\beta$, the diffusion tensor $A$, and the penalty parameter $\gamma$.

Remark 1. As proved in [22, Theorem 6.1 and Corollary 5.19], the exponential convergence result (32) also holds for spaces with uniform and isotropic polynomial degrees, i.e., for the family

$$
V_{\sigma}^{\ell}=S^{p^{(\ell)}}\left(\mathscr{M}_{\sigma}^{(\ell)}\right), \quad \ell \geq 1,
$$

provided that $p^{(\ell)} \simeq \ell$. However, in this case, the constant $b$ in the exponent has to be replaced by a smaller value $\bar{b}>0$.

\subsection{Ingredients of the proof}

Let us give some insights into the proof of Theorem 2. We apply the error estimates of Theorem 1. To that end and according to (23), we subdivide the geometric mesh $\mathscr{M}_{\sigma}^{(\ell)}$ into 


$$
\mathscr{M}_{\sigma}^{(\ell)}=\mathfrak{O}_{\sigma}^{(\ell)} \dot{\cup} \mathfrak{T}_{\sigma}^{(\ell)} .
$$

After specification of the $h p$-version interpolation operator $\Pi u$ in (24), Theorem 1 requires bounding the two terms $E_{\mathfrak{O}_{\sigma}^{(\ell)}}[\eta]$ and $E_{\mathfrak{T}_{\sigma}^{(\ell)}}[\eta]$ in (27) and (28), respectively. Since the approximation spaces are discontinuous, we can choose different interpolation operators in the two submeshes $\mathfrak{O}_{\sigma}^{(\ell)}$ and $\mathfrak{T}_{\sigma}^{(\ell)}$.

Bounding $E_{\mathfrak{D}_{\sigma}^{(\ell)}}[\eta]$ : In the elements away from $\mathscr{S}$, we choose $\Pi u$ to be an elementwise tensorized operator of univariate $h p$-interpolation operators: for an element $K \in \mathfrak{O}_{\sigma}^{(\ell)}$ and a polynomial degree $\underline{p}_{K}=\left(p_{1}, p_{2}, p_{3}\right)$, we set

$$
\left.(\Pi u)\right|_{K}=\left.\pi_{p_{1}, 2}^{1} \otimes \pi_{p_{2}, 2}^{2} \otimes \pi_{p_{3}, 2}^{3} u\right|_{K}, \quad K \in \mathfrak{O}_{\sigma}^{(\ell)},
$$

where $\pi_{p_{i}, 2}^{i}$ is a properly scaled version of the $C^{1}$-conforming univariate projector into polynomials of degree $p_{i}$, constructed and analyzed in [6, Section 8] and acting in coordinate direction $x_{i}$.

To take into account different weighting of the singularities in the different neighborhoods, we shall further subdivide $\mathfrak{O}_{\sigma}^{(\ell)}$ into discrete corner, edge, corner-edge and interior neighborhoods of the form

$$
\mathfrak{O}_{\sigma}^{\ell}=\mathfrak{O}_{\mathscr{C}}^{\ell} \dot{\cup} \mathfrak{O}_{\mathscr{E}}^{\ell} \dot{\cup} \mathfrak{O}_{\mathscr{C} \mathscr{E}}^{\ell} \dot{\cup} \mathfrak{O}_{\text {int }}^{\ell} .
$$

In each of these neighborhoods, the geometrically refined elements can be grouped into certain subsets of elements with identical scaling properties in terms of their relative distance to the sets $\mathscr{C}$ and $\mathscr{E}$. Hence, combining the analytic regularity properties in each of the discrete neighborhoods with classical $h p$-approximation techniques for $u-\Pi u$ (similarly to the two-dimensional case) yields

$$
E_{\mathfrak{O}_{\sigma}^{(\ell)}}[\eta] \leq C \exp (-2 b \ell),
$$

with constants $C>0$ and $b>0$ independent of $\ell$. We refer to [22, Sections 5.2 and 5.3] for details.

Bounding $E_{\mathfrak{T}_{\sigma}^{(\ell)}}[\eta]$ : For elements $K \in \mathfrak{T}_{\sigma}^{(\ell)}$ at the boundary of $\Omega$, the zero interpolation operator $\Pi u \equiv 0$ is sufficient; indeed, this may be motivated by the fact that the exact solution $u$ satisfies homogeneous Dirichlet boundary conditions. In addition, the weights appearing in the $\|\cdot\|_{M_{-1-\underline{\beta}}^{2}}$-norm from (9) carry negative exponents for $|\alpha|=0,1$, which results in exponentially small scaled element contributions in $\mathfrak{T}_{\sigma}^{(\ell)}$. Thence, the following bound can be obtained:

$$
E_{\mathfrak{T}_{\sigma}^{(\ell)}}[\eta] \leq C \exp (-2 b \ell)
$$

with constants $C>0$ and $b>0$ independent of $\ell$, see [22, Section 5.4]. 
Counting the degrees of freedom: From Theorem 1 and the bounds (37), (38), we conclude that

$$
\left\|u-u_{\mathrm{DG}}\right\|_{\mathrm{DG}} \leq C \exp (-b \ell) .
$$

Then we note that $N=\operatorname{dim}\left(V_{\sigma, \mathfrak{s}}^{(\ell)}\right) \simeq b \ell^{5}+O\left(\ell^{4}\right)$ for $\ell \rightarrow \infty$, which implies the bound (32).

\section{Numerical experiments}

For the numerical experiments the software library deal.ii [4] is employed. Our computations are based on the geometrically (with ratio $\sigma=1 / 2$ ) refined $h p$ spaces $V_{\sigma}^{\ell}$ from (33) featuring uniform and isotropic polynomial degree $p \simeq \ell$, where $\ell$ is the number of mesh layers. We test the $h p$-DGFEM (16) for the three reference situations 'corner patch', 'edge patch', 'corner-edge patch', as displayed in Figure 1 (and scaled to the unit cube $\left.(0,1)^{3}\right)$. They correspond to the $h p$-extensions $(E x 2),(E x 3),(E x 4)$ in [21], respectively. In all experiments, the penalty parameter in (17) is chosen to be $\gamma=10$. Then, we monitor the decay of the error measured in the DG-energy norm (19) as the number of refinements $\ell$ is increased. In all experiments we prescribe the exact solution $u$, and choose the right-hand side $f$ in (1) (as well as the (nonhomogeneous) Dirichlet boundary conditions) accordingly.

- Corner patch (Ex2): The exact solution is chosen to be $u_{c}\left(r_{c}\right)=r_{c}^{\gamma_{c}}$, with $\gamma_{c}=1 / 3$, where $r_{c}$ denotes the distance to the origin. This solution has an isotropic singularity at 0 , and is resolved using the isotropic geometric corner mesh shown in Figure 1 (left). The number of degrees of freedom $N$ in the corresponding $h p$-spaces is proportional to $\ell^{4} \simeq p^{4}$. In Figure 2, we observe that the DG energy error decays with a nearly constant slope in a semi-logarithmic plot, thereby confirming exponential convergence (with respect to $N^{1 / 4}$ ).

- Edge patch (Ex3): Here, we choose $u_{e}\left(r_{e}\right)=r_{e}^{\gamma_{e}}$, for $\gamma_{e}=1 / 2$, with $r_{e}$ signifying the distance to the edge $e=\left\{x_{1}=0\right\} \times\left\{x_{2}=0\right\} \times\left\{0<x_{3}<1\right\}$. The solution exhibits an anisotropic and non-local edge singularity along $e$, and is refined by means of the anisotropic geometric edge-mesh depicted in Figure 1 (center). Again, the number of degrees of freedom is proportional to $\ell^{4} \simeq p^{4}$, and the exponential decay of the DG energy error is clearly visible in Figure 2.

- Corner-edge patch (Ex4): Finally, we consider the anisotropic corner-edge singularity solution $u_{c e}\left(r_{c}, r_{e}\right)=r_{c}^{\gamma_{c}} r_{e}^{\gamma_{e}}$, with $\gamma_{c}=1 / 3$ and $\gamma_{e}=1 / 2$. It is refined by employing the anisotropic corner-edge mesh presented in Figure 1 (right). This is the most complex of the three model cases discussed here; in fact, in contrast to the previous examples, it features $N \simeq \ell^{5} \simeq p^{5}$ degrees of freedom. Correspondingly, the DG energy error is plotted against the fifth root of $N$. As before, exponential convergence is achieved already after a few initial refinements.

Our experiments show that the $h p$-DGFEM (16) on the proposed geometric $h p$ meshes is able to resolve isotropic as well as anisotropic singularities, and, in par- 


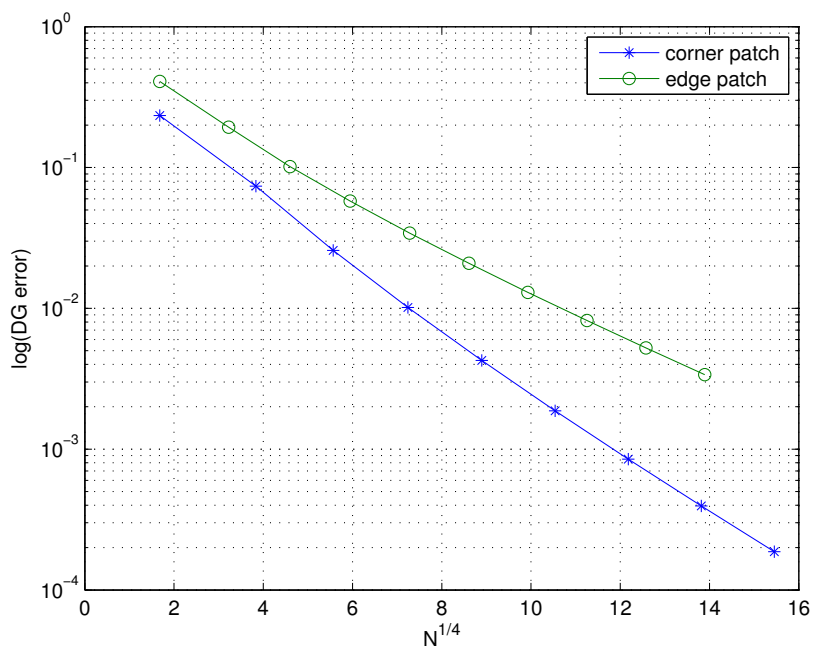

Fig. 2 Performance of the $h p$-DGFEM in corner and edge patches.

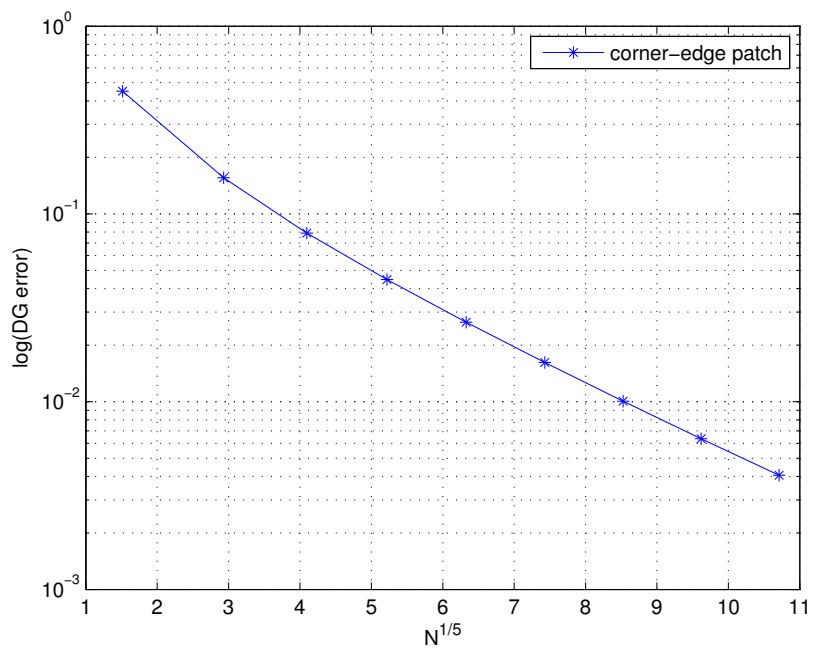

Fig. 3 Performance of the $h p$-DGFEM in corner-edge patch.

ticular, that exponential rates of convergence are attained in all the reference configurations shown in Figure 1. 
$h p$-DGFEM for elliptic problems in polyhedral domains

\section{Concluding remarks}

Ongoing research is concerned with extensions of the exponential convergence theory for $h p$-DGFEM in three dimensions to elliptic problems with mixed and Neumann boundary conditions (which are considerably more involved than the homogeneous Dirichlet boundary conditions considered in this paper), to problems with more complicated geometries and non-constant coefficients, as well as to more general elliptic systems.

Acknowledgements This work was supported in part by the Natural Sciences and Engineering Research Council of Canada (NSERC), the European Research Council AdG grant STAHDPDE 247277, and the Swiss National Science Foundation (SNF, Grant 200020_144442/1).

\section{References}

1. D. N. Arnold, F. Brezzi, B. Cockburn, and L. D. Marini. Unified analysis of discontinuous Galerkin methods for elliptic problems. SIAM J. Numer. Anal., 39:1749-1779, 2001.

2. I. Babuška and B. Q. Guo. Regularity of the solution of elliptic problems with piecewise analytic data. I. Boundary value problems for linear elliptic equation of second order. SIAM J. Math. Anal., 19(1):172-203, 1988.

3. I. Babuška and B. Q. Guo. Approximation properties of the $h$ - $p$ version of the finite element method. Comput. Methods Appl. Mech. Engrg., 133(3-4):319-346, 1996.

4. W. Bangerth, R. Hartmann, and G. Kanschat. deal.II - a general purpose object oriented finite element library. ACM Trans. Math. Softw., 33(4):24/1-24/27, 2007.

5. M. Costabel, M. Dauge, and S. Nicaise. Analytic regularity for linear elliptic systems in polygons and polyhedra. Math. Models Methods Appl. Sci., 22(8), 2012.

6. M. Costabel, M. Dauge, and C. Schwab. Exponential convergence of $h p$-FEM for Maxwell's equations with weighted regularization in polygonal domains. Math. Models Methods Appl. Sci., 15(4):575-622, 2005.

7. W. Gui and I. Babuška. The $h, p$ and $h-p$ versions of the finite element method in 1 dimension. II. The error analysis of the $h$ - and $h$ - $p$ versions. Numer. Math., 49(6):613-657, 1986.

8. B. Q. Guo. The $h-p$ version of the finite element method for elliptic equations of order $2 m$. Numer. Math., 53(1-2):199-224, 1988.

9. B. Q. Guo. The $h-p$ version of the finite element method for solving boundary value problems in polyhedral domains. In Boundary Value Problems and Integral Equations in Nonsmooth Domains, volume 167 of Lecture Notes in Pure and Applied Mathematics, pages 101-120. Dekker, New York, 1995.

10. B. Q. Guo and I. Babuška. The $h p$-version of the finite element method. Part I: The basic approximation results. Comp. Mech., 1:21-41, 1986.

11. B. Q. Guo and I. Babuška. The $h p$-version of the finite element method. Part II: General results and applications. Comp. Mech., 1:203-220, 1986.

12. B. Q. Guo and I. Babuška. Regularity of the solutions for elliptic problems on nonsmooth domains in $\mathbb{R}^{3}$. I. Countably normed spaces on polyhedral domains. Proc. Roy. Soc. Edinburgh Sect. A, 127(1):77-126, 1997.

13. B. Q. Guo and I. Babuška. Regularity of the solutions for elliptic problems on nonsmooth domains in $\mathbb{R}^{3}$. II. Regularity in neighbourhoods of edges. Proc. Roy. Soc. Edinburgh Sect. A, 127(3):517-545, 1997.

14. B. Q. Guo and C. Schwab. Analytic regularity of Stokes flow on polygonal domains in countably weighted Sobolev spaces. J. Comp. Appl. Math., 119:487-519, 2006. 
15. P. Houston, C. Schwab, and E. Süli. Stabilized $h p$-finite element methods for first-order hyperbolic problems. SIAM J. Numer. Anal., 37:1618-1643, 2000.

16. P. Houston, C. Schwab, and E. Süli. Discontinuous $h p$-finite element methods for advectiondiffusion-reaction problems. SIAM J. Numer. Anal., 39:2133-2163, 2002.

17. I. Perugia and D. Schötzau. An $h p$-analysis of the local discontinuous Galerkin method for diffusion problems. J. Sci. Comput., 17:561-571, 2002.

18. D. Schötzau and C. Schwab. Exponential convergence in a Galerkin least squares $h p$-FEM for Stokes flow. IMA J. Numer. Anal., 21:53-80, 2001.

19. D. Schötzau, C. Schwab, and A. Toselli. Stabilized $h p$-DGFEM for incompressible flow. Math. Models Methods Appl. Sci., 13(10):1413-1436, 2003.

20. D. Schötzau, C. Schwab, and A. Toselli. Mixed $h p$-DGFEM for incompressible flows. II. Geometric edge meshes. IMA J. Numer. Anal., 24(2):273-308, 2004.

21. D. Schötzau, C. Schwab, and T. P. Wihler. $h p$-dGFEM for elliptic problems in polyhedra. I: Stability and quasioptimality on geometric meshes. Technical Report 2009-28 (revised January 2012), Seminar for Applied Mathematics, ETH Zürich, 2009.

22. D. Schötzau, C. Schwab, and T. P. Wihler. $h p$-dGFEM for elliptic problems in polyhedra. II: Exponential convergence. Technical Report 2009-29 (revised January 2012), Seminar for Applied Mathematics, ETH Zürich, 2009.

23. D. Schötzau and T. P. Wihler. Exponential convergence of mixed $h p$-DGFEM for Stokes flow in polygons. Numer. Math., 96:339-361, 2003.

24. T. P. Wihler, P. Frauenfelder, and C. Schwab. Exponential convergence of the $h p$-DGFEM for diffusion problems. Comput. Math. Appl., 46:183-205, 2003.

25. T. P. Wihler and M. Wirz. Mixed $h p$-Discontinuous Galerkin FEM for linear elasticity in three dimensions. Math. Models Methods Appl. Sci., 22(8), 2012. 